\title{
Braune Getränke und bunte Pillen - zur Abhängigkeit der Fachgesellschaften von Industriegeldern
}

Jürg Nadig

Präsident der Schweizerischen Gesellschaft für Medizinische Onkologie
1 Marti C. ... der werfe den ersten Stein. Schweiz Ärztezeitung. 2011;92(1/2):39.

\section{Korrespondenz:}

Dr. med. Jürg Nadig MAE Facharzt für Medizinische Onkologie

und Innere Medizin FMH

Bannhaldenstrasse 7

CH-8180 Bülach

juerg.nadig@hin.ch

\section{Lieber Christian}

Spruchbänder und Plakatwände beim Brandenburger Tor, mit denen Coca-Cola während des Kongresses der Deutschen Gesellschaft für Hämatologie und Onkologie (DGHO) den Tag zur Deutschen Einheit präsentierte, regten mich anlässlich des Gesellschaftsabends des Kongresses zu einer geschichtsphilosophischen Betrachtung an. Die Spruchbänder erinnerten mich an den Wettlauf zum Mond: Auf einem Cartoon im Nebel-

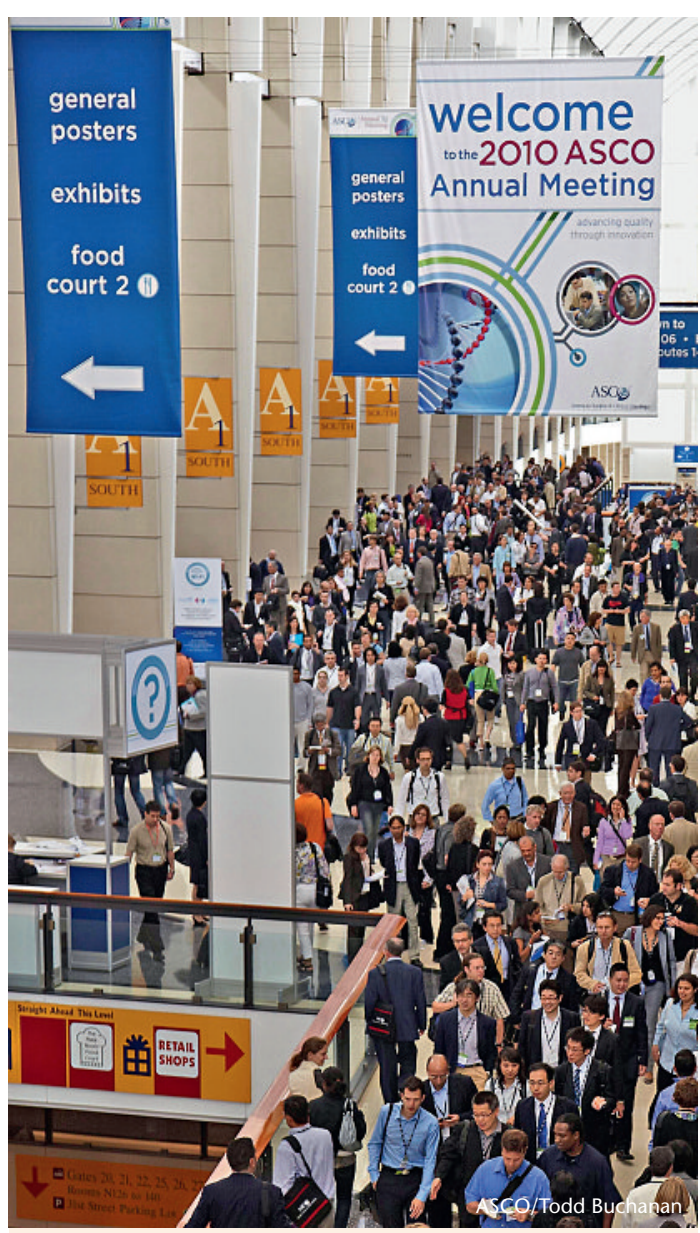

Weltweit erhalten medizinische Fachgesellschaften bei Kongressen Gelder industrieller Aussteller für die Verpachtung von Werberechten. spalter malten die sowjetischen Kosmonauten den Mond rot an, als sie ihn als erste betraten. Die später kommenden US-amerikanischen Astronauten schrieben in die rote Mondscheibe mit weisser Farbe «CocaCola». Die Frage des Sponsorings des DGHO-Kongresses war in diesem Zusammenhang kein Thema. Dein Artikel gibt mir aber die Möglichkeit, mich als Präsident einer der mitgestaltenden Gesellschaften zum Thema der Abhängigkeit der Fachgesellschaften von Industriegeldern öffentlich zu äussern.

SAMW-Richtlinien sollten die Zusammenarbeit von Ärzteschaft und Industrie regeln. Aber auch wenn diese eingehalten werden, die Gelder in einen Pool fliessen und der wissenschaftliche und der kommerzielle Teil von Kongressen organisatorisch und räumlich getrennt sind, wie dies in Berlin dank der Grösse des Kongresszentrums möglich war, erhalten letztlich die Fachgesellschaften Gelder von den Ausstellern für die Verpachtung von Werberechten. Die Fachgesellschaft kann damit Aufgaben finanzieren, die ihr von der Politik und der FMH übertragen, aber sonst nicht finanziert werden (Tariffragen, Tarifentwicklung, Mitarbeit bei DRG-Gestaltung, Weiter- und Fortbildung, Off label use, Register zur Qualitätserfassung). Diese Aufgaben nahmen in den letzten Jahren stetig zu.

Um die Milizionäre im Vorstand zu entlasten, erwogen wir eine Professionalisierung der Vereinsarbeit mit einem Sekretär und einem Sekretariat. Ein externer Berater schätzte den finanziellen Aufwand auf 200000 Franken pro Jahr. Wir hätten den Mitgliederbeitrag auf 1000 Franken erhöhen müssen. Rechnet man die Mitgliederbeiträge in den anderen standespolitischen Organisationen zusammen, entspricht das 2-3\% des Referenzeinkommens. Darin nicht eingerechnet sind die zusätzlichen Kosten für die Pflege von Behandlungsregistern als Qualitätsinstrument oder andere qualitätssichernde Massnahmen in der Medizinischen Onkologie. Diese Kosten können aber nicht auf die Preise abgewälzt werden, da die Preise im Tarif reguliert sind.

In Deutschland wird der Kongressgewinn zur Subvention der Mitgliederbeiträge und der Kongresseintritte verwendet. Kostendeckende Beiträge führten zu einem Schrumpfen der Mitgliederzahl und zu einer Ausweitung der Gratisfortbildungen der Industrie. Lei- 
der gibt es auch in der Schweiz immer mehr Fachärzte, die ihr Einkommen optimieren, indem sie weder Mitglied schweizerischer oder internationaler Fachgesellschaften sind noch der FMH angehören. Geschäftstüchtig können sie so in ihrer Ich-AG mehrere tausend Franken sparen und ihre Kostenstruktur optimieren. Den kleinen Fachgesellschaften gehen dadurch nicht nur Mitgliederbeiträge verloren. Es fehlen auch Kollegen, die sich standespolitisch einsetzen.

Welche Alternative der Finanzierung der öffentlichen Aufgaben der Fachgesellschaften gäbe es? Statt über die Margensenkung bei den Medikamenten Kosten zu sparen, könnte mit einem fixen Prozentsatz auf den Fabrikabgabepreis die Weiter- und Fortbildung sowie die Qualitätssicherung, verbunden mit Begleit- und Versorgungsforschung, finanziert werden. Damit könnte zumindest ein Teil der Werbekosten, über die heute die Pharmafirmen verfügen, wieder in den öffentlichen Raum zurückgeführt und kontrolliert werden, sind doch die Medikamente über eine Zwangsabgabe (Krankenkassenprämie) finanziert. Damit wäre eine grössere Unabhängigkeit der Fachgesellschaften gegeben.

Herzliche Grüsse Jürg 